\title{
Facilitating Planning by using Self-organization
}

\author{
Oliver Kosak \\ Supervisor: Wolfgang Reif \\ Institute for Software \& Systems Engineering, Augsburg University, Germany \\ E-Mail: $\{$ kosak, reif $\} @ i$ isse.de
}

\begin{abstract}
Current approaches to increase autonomy of multirobot applications are often tailored to particular applications. This specialization is necessary due to specific requirements to the robots' capabilities in dedicated applications or environments. We present an approach to overcome this specialization by separating capabilities from robots and enabling their selforganized reconfiguration at run-time. This reduces the need of re-planning by absorbing uncertainties and at the same time enables to easily use the same robots in different scenarios.
\end{abstract}

Index Terms-MAS; SAR; Planning; Self-optimization;

\section{Motivation}

Mobile multi-robot systems gain increasing attention in research and industry. While applications in the domain of Search and Rescue (SAR) have profited from these developments for years (e.g., human-robot-interaction in alpine $S A R$ scenarios [1], or wilderness SAR [2]), the potential of multirobot applications now is recognized in many other domains. These include environmental research with unmanned underwater [3], [4] or surface vehicles [5], distributed surveillance of critical infrastructure [6], [7], [8], or dealing with major catastrophes (e.g., chemical accidents) [9], among others. We identified common steps relevant in all of these applications and call them tasks of ScORe missions [10]. Given, e.g., a chemical accident, the situation has 1) to be assessed by searching $(S)$ for the relevant harmful substance with as much robots equipped with a universal gas sensor as possible. Within a subsequent task, the dissemination of the identified substance has 2) to be continuously observed $(\mathrm{cO})$ by robots equipped with specialized sensors as well as robots capable of estimating local weather conditions. As can be seen, the distribution of capabilities on robots is highly specific to environmental conditions and might change over time. Observations may trigger 3) appropriate reactions ( $R e$ ), e.g., the evacuation of inhabited areas, done by robots equipped with appropriate actuators (e.g., sirens) that has to be done in parallel to the $c O$ task. In all tasks, robots have to be able to fly. Existing approaches for coordinating and controlling multiple robots in ScORe applications can roughly be divided into two groups. On the one hand, there are the so-called swarm robotics approaches [3], [5], [11], where great numbers of homogeneous robots are used to collectively achieve a common goal through emergent, fault-tolerant behavior. Such algorithms are very useful to robustly solve special tasks (i.e., single tasks of ScORe missions), like finding the highest parameter concentration with particle-swarm optimization techniques [12], finding consensus over multiple options [13], [14], clustering [5], or collectively transporting objects [15], [16], among others. But as theses approaches often lack a sophisticated decision logic, their ability to deal with complex tasks is limited [17], as a swarm has no clue what comes next after achieving its current goal. Thus, in the context of ScORe missions, swarm robotic approaches are not capable of autonomously switching from one phase to another, nor to a different ScORe mission. On the other hand, approaches that focus on combining the special abilities of few, heterogeneous robots for reaching a goal are often explicitly designed for a certain application or environment (e.g., searching or retrieving an object in an indoor environment [18], supporting human guided SAR missions in alpine [1] or wilderness [2] environments). While the proposed solutions deliver promising results for their dedicated applications, they lack the flexibility to easily adapt to slightly different scenarios (different ScORe missions), as they are highly specialized towards the use case they have been designed for, with regards to software [18], [19] as well as hardware [15], [20]. This at least is the case when the adapted scenario requires different capabilities from participating robots, e.g., switching from chemical accident handling to environmental monitoring. Another deficiency current approaches for ScORe missions suffer from is their way of handling uncertainties. These typically occur during execution within a real world environment [21]. Solutions like online re-planning [22] are time-intensive and comprehensive as they require details for multiple agents (e.g., multi-agent planning [23] or [24]).

\section{Objectives}

We envision an ensemble of cooperative robots consisting of several modular hardware parts, re-combinable at run-time. These will contain actuators like quadrocopters and mobile ground units as well as different sensors. Hardware modules are assumed to be self-aware and easily exchangeable, which will be achieved by an accompanying dissertation. For terms of simplification, we assume to have a reliable WiFi network for communication purposes, e.g., achieved through techniques developed in other projects like [25], [26]. The main objective of this dissertation is to develop a reference system architecture and distributed algorithms for ScORe applications. The goal is to enable the dynamical re-configuration of robots' capabilities within 
the ensemble in a self-organized fashion by exchangeable, self aware hardware modules. Thereby robust execution of ScORe missions will be enabled, absorbing uncertainties typically occurring in this context (e.g., loss of components, changing $\mathrm{ScORe}$ task requirements). This will facilitate the planning process for ScORe missions, where actions of single robots no longer have to be planned in detail, but only actions of the whole ensemble. Planned task only have to guarantee the general feasibility of each planned ScORe task, independent of the concrete assignment of tasks to robots as well as of the concrete assignment of capabilities to robots. Assignments on both levels will be calculated and executed by the ensemble itself in a distributed and self-organized fashion, respecting all low-level constraints concerning the availability of capabilities in certain hardware configurations for each robot. This approach will enable the system to adapt to frequently changing situations that typically occur in ScORe applications (i.e., changing tasks or even changing missions) and separate this problem from the planning problem. By establishing this system architecture, we will overcome the specialization issue of current approaches (cf. Sect. 1). For being able to instruct the ensemble with $\mathrm{ScORe}$ missions, a task and capability formalism has to be found. This formalism has to support task decomposition and planning in an abstract manner, regarding only the ensemble as a whole as well as generally available abilities of the ensemble in one of its possible configurations (multi-robot coalitions, single-robot capabilities), respecting user defined time or quality constraints. Ensemble abilities are meant to be self-organization mechanism that can be used to solve ScORe tasks (e.g., Search can be performed with an adapted particle swarm algorithm [12]). Robot abilities result from certain capability configurations and are meant to indicate if a robot is able to participate in such an ensemble mechanism. This approach should ease handling uncertainties in real-world environments that typically increase the complexity of planning.

\section{Methodology}

To reach the goals described in Sect. 2, we will adopt and extend a broad field of existing technologies and methodologies in an iterative development process. For appropriately realizing the ScORe reference system architecture and for managing the dynamic availability of capabilities at runtime, we plan to build on the multi-agent framework Jadex [27], which offers possibilities for cross-device distribution of algorithms and communication between them. Robots as well as their capabilities and functionalities will be represented by agents within this framework. The architecture of the system will be structured into layers to enable planning of a ScORe mission on ensemble level, and avoid detailed, agent-level planning. Therefore, we plan to adopt existing approaches for multi-robot task definition like [22], [28], [29], [30], enriched with an abstract robot- and ensembleability description formalism on the topmost task layer. On a second ensemble algorithm layer, we plan to integrate self-organization algorithm adaptations, that fit the ScORe phases context, e.g., particle swarm optimization [12] or

\begin{tabular}{l|lll}
$\#$ & Workpackage & Start & End \\
\hline 1 & architecture design and implementation & Running & Q4 2019 \\
\hline 2 & ScORe definition language evaluations & Q1 2018 & Q4 2019 \\
\hline 3 & simple ScORe mission handling & Running & Q1 2018 \\
\hline 4 & ScORe including reconfigurations & Q2 2018 & Q4 2018 \\
\hline 5 & resource handling MR-MT & Q1 2019 & Q4 2019 \\
\hline 6 & real-world experiment & Q1 2020 & Q3 2020 \\
& TABLE 1. WOR KING PLAN FOR THE NEXT THREE YEARS.
\end{tabular}

coverage planning [31] for search, or guided collective movement (boiding/flocking) [32], [33], [34] for navigation, able to solve tasks from the task layer. The required robot behavior for participating in these ensemble layer algorithms is located on a third agent layer whose execution may be possible or not, depending on the robots' current capabilities. Capabilities result from the combination of attached hardware modules, their availability is calculated in a fourth capability layer. To decide which agents fit best to the requirements of an ScORe task on ensemble layer, we will adopt appropriate distributed task allocation approaches, e.g., market-based task allocation [35]. To resolve situations, where task requirements cannot be met with the current robot/ensemble configuration, we will use distributed constraint optimization technologies [36] to collectively decide which configuration is capable of solving the current task. For our real-world evaluations we will use the RoboticsAPI [37] for concrete hardware control as well as simulation. We see great potential in combining planning with selforganization in the proposed way. Because single agent actions no longer have to be planned explicitly and failurestates can be resolved by re-configuring the system, the need for re-planning is heavily reduced. We will evaluate this thesis by comparing our approach against state of the art multi-agent task planners like [29], [30] to determine the level of complexity each approach is suitable for.

\section{Research Plan}

We already gained experience in handling ScORe missions [38], [39], robust and distributed multi-agent task allocation [40], developed and evaluated a first version of our proposed architecture [10], [39], and investigated current challenges and perspectives of self-organizing systems [41]. Our architecture will be iteratively improved throughout the dissertation project. Its performance against other solutions will be frequently evaluated in its particular state of extension, starting with 1) simple ScORe missions without the need of capability re-configurations, continuing with 2) ScORe missions that need adaptation of the system's configuration normally requiring re-planning, and finaly 3) extending the capability concept to enable MR-MT [42] handling for ScORe missions. In addition to that, we will evaluate appropriate formalisms for ScORe task definition in each state of the iterative process, which will be done by extending current techniques suitable for our proposed approach. All of our results will be evaluated iteratively in simulation as well as in a real-world scenario, that constitutes a simplified version of the ScORe scenario introduced in Sect. 1 including multiple robots as well as reconfigurable hardware modules. Tab. 1 shows a chronological schedule of packages portioning the proposed workload. 


\section{References}

[1] L. Marconi, S. Leutenegger, S. Lynen, M. Burri, R. Naldi, and C. Melchiorri, "Ground and aerial robots as an aid to alpine search and rescue: Initial sherpa outcomes," in Safety, Security, and Rescue Robotics (SSRR), 2013 IEEE Int. symposium on. IEEE, 2013, pp. $1-2$.

[2] E. F. Flushing, L. M. Gambardella, and G. A. D. Caro, "A mathematical programming approach to collaborative missions with heterogeneous teams," in 2014 IEEE/RSJ Int. Conf. on Intelligent Robots and Systems, Sept 2014, pp. 396-403.

[3] T. Schmickl, R. Thenius, C. Moslinger, J. Timmis, A. Tyrrell, M. Read, J. Hilder, J. Halloy, A. Campo, C. Stefanini, L. Manfredi, S. Orofino, S. Kernbach, T. Dipper, and D. Sutantyo, "Cocoro - the self-aware underwater swarm," in 2011 Fifth IEEE Conference on Self-Adaptive and Self-Organizing Systems Workshops, Oct 2011, pp. $120-126$.

[4] R. Thenius, D. Moser, S. Kernbach, I. Kuksin, O. Kernbach, E. Elena Kuksina, N. Miśković, S. Bogdan, T. Petrović, A. Babić, F. Boyer, V. Lebastard, S. Bazeille, G. W. Ferrari, E. Donati, R. Pelliccia, D. Romano, C. Stefanini, M. Morgantin, A. Campo, and T. Schmickl, "subclutron: a learning, self-regulating, self-sustaining underwater society/culture of robots," Art. Life and Intelligent Agents Symposium, 2016, 2016.

[5] M. Duarte, V. Costa, J. Gomes, T. Rodrigues, F. Silva, S. M. Oliveira, and A. L. Christensen, "Evolution of collective behaviors for a real swarm of aquatic surface robots," PLOS ONE, vol. 11, no. 3, pp. 1-25, 03 2016. [Online]. Available: http: //dx.doi.org/10.1371\%2Fjournal.pone.0151834

[6] J. Zhang, L. Liu, B. Wang, X. Chen, Q. Wang, and T. Zheng, "High speed automatic power line detection and tracking for a uav-based inspection," in 2012 International Conference on Industrial Control and Electronics Engineering, Aug 2012, pp. 266-269.

[7] C. Martinez, C. Sampedro, A. Chauhan, and P. Campoy, "Towards autonomous detection and tracking of electric towers for aerial power line inspection," in 2014 International Conference on Unmanned Aircraft Systems (ICUAS), May 2014, pp. 284-295.

[8] L. Matikainen, M. Lehtomki, E. Ahokas, J. Hyypp, M. Karjalainen, A. Jaakkola, A. Kukko, and T. Heinonen, "Remote sensing methods for power line corridor surveys," $\{$ ISPRS $\}$ Journal of Photogrammetry and Remote Sensing, vol. 119, pp. 10 - 31, 2016. [Online]. Available: http://www.sciencedirect.com/science/article/pii/ S0924271616300697

[9] K. Daniel, B. Dusza, A. Lewandowski, and C. Wietfelds, "Airshield: A system-of-systems muav remote sensing architecture for disaster response," in Proc. 3rd Annual IEEE Systems Conf. (SysCon), 2009.

[10] O. Kosak, C. Wanninger, A. Angerer, A. Hoffmann, A. Schiendorfer, and H. Seebach, "Towards self-organizing swarms of reconfigurable self-aware robots," in Found. and Applications of Self* Systems, IEEE Int. Workshops on. IEEE, 2016, pp. 204-209.

[11] D. Caron, B. Stauffer, L. Darjany, C. Oberg, A. Pereira, J. Das, H. Heidarsson, R. Smith, E. Smith, E. Seubert et al., "Networked aquatic microbial observing systems: An overview," Center for Embedded Network Sensing, 2009.

[12] Y. Zhang, S. Wang, and G. Ji, "A comprehensive survey on particle swarm optimization algorithm and its applications," Mathematical Problems in Engineering, vol. 2015, 2015.

[13] H. Hamann, "Towards swarm calculus: urn models of collective decisions and universal properties of swarm performance," Swarm Intelligence, vol. 7 , no. 2, pp. 145-172, 2013. [Online]. Available: http://dx.doi.org/10.1007/s11721-013-0080-0

[14] G. Valentini, H. Hamann, and M. Dorigo, "Efficient decision-making in a self-organizing robot swarm: On the speed versus accuracy trade-off," in Proc. of the 2015 Int. Conf. on Autonomous Agents and Multiagent Systems, ser. AAMAS '15. Richland, SC: Int. Found. for Autonomous Agents and Multiagent Systems, 2015, pp. 1305-1314. [Online]. Available: http://dl.acm.org/citation.cfm? id $=2772879.2773319$
[15] M. Dorigo, E. Tuci, R. Groß, V. Trianni, T. H. Labella, S. Nouyan, C. Ampatzis, J.-L. Deneubourg, G. Baldassarre, S. Nolfi et al., "The swarm-bots project," in Int. Workshop on Swarm Robotics. Springer, 2004, pp. 31-44.

[16] R. Gross and M. Dorigo, "Towards group transport by swarms of robots," International Journal of Bio-Inspired Computation, vol. 1, no. 1-2, pp. 1-13, 2009.

[17] M. Brambilla, E. Ferrante, M. Birattari, and M. Dorigo, "Swarm robotics: a review from the swarm engineering perspective," Swarm Intelligence, vol. 7, no. 1, pp. 1-41, 2013.

[18] M. Dorigo, D. Floreano, L. M. Gambardella, F. Mondada, S. Nolfi, T. Baaboura, M. Birattari, M. Bonani, M. Brambilla, A. Brutschy, D. Burnier, A. Campo, A. L. Christensen, A. Decugniere, G. D. Caro, F. Ducatelle, E. Ferrante, A. Forster, J. M. Gonzales, J. Guzzi, V. Longchamp, S. Magnenat, N. Mathews, M. M. de Oca, R. O'Grady, C. Pinciroli, G. Pini, P. Retornaz, J. Roberts, V. Sperati, T. Stirling, A. Stranieri, T. Stutzle, V. Trianni, E. Tuci, A. E. Turgut, and F. Vaussard, "Swarmanoid: A novel concept for the study of heterogeneous robotic swarms," IEEE RAM, vol. 20, no. 4, pp. 60-71, 2013.

[19] J. Scherer, S. Yahyanejad, S. Hayat, E. Yanmaz, T. Andre, A. Khan, V. Vukadinovic, C. Bettstetter, H. Hellwagner, and B. Rinner, "An autonomous multi-uav system for search and rescue," in Proc. of the First Workshop on Micro Aerial Vehicle Networks, Systems, and Applications for Civilian Use, ser. DroNet '15. New York, NY, USA: ACM, 2015, pp. 33-38. [Online]. Available: http://doi.acm.org/10.1145/2750675.2750683

[20] S. Mintchev, E. Donati, S. Marrazza, and C. Stefanini, "Mechatronic design of a miniature underwater robot for swarm operations," in 2014 IEEE Int. Conf. on Robotics and Automation (ICRA). IEEE, 2014, pp. 2938-2943.

[21] S. Magnenat, J.-C. Chappelier, and F. Mondada, "Integration of online learning into htn planning for robotic tasks." in AAAI Spring Symposium: Designing Intelligent Robots, 2012.

[22] S. Koenig, "Agent-centered search," AI Magazine, vol. 22, no. 4, p. 109, 2001.

[23] D. V. Pynadath and M. Tambe, "An automated teamwork infrastructure for heterogeneous software agents and humans," Autonomous Agents and Multi-Agent Systems, vol. 7, no. 1, pp. 71-100, 2003. [Online]. Available: http://dx.doi.org/10.1023/A: 1024176820874

[24] R. I. Brafman and C. Domshlak, "From one to many: Planning for loosely coupled multi-agent systems," in ICAPS, 2008, pp. 28-35.

[25] K. Daniel, S. Rohde, and C. Wietfeld, "Leveraging public wireless communication infrastructures for uav-based sensor networks," in 2010 IEEE Int. Conf. on Technologies for Homeland Security (HST), Nov 2010, pp. 179-184.

[26] Self-organizing intelligent network of uavs. http://uav.lakesidelabs.com/overview/sinus/. [Online]. Available: http://uav. lakeside-labs.com/overview/sinus/

[27] L. Braubach and A. Pokahr, "Developing distributed systems with active components and jadex," Scalable Computing: Practice and Experience, vol. 13, no. 2, pp. 100-120, 2012.

[28] F. Amigoni, N. Gatti, C. Pinciroli, and M. Roveri, "What planner for ambient intelligence applications?" IEEE Trans. on Systems, Man, and Cybernetics - Part A: Systems and Humans, vol. 35, no. 1, pp. 7-21, Jan 2005.

[29] H. Skubch, M. Wagner, R. Reichle, S. Triller, and K. Geihs, "Towards a comprehensive teamwork model for highly dynamic domains." in ICAART (2), 2010, pp. 121-127.

[30] I. Georgievski and M. Aiello, "An overview of hierarchical task network planning," arXiv preprint arXiv:1403.7426, 2014.

[31] A. Breitenmoser, M. Schwager, J. C. Metzger, R. Siegwart, and D. Rus, "Voronoi coverage of non-convex environments with a group of networked robots," in 2010 IEEE International Conference on Robotics and Automation, May 2010, pp. 4982-4989. 
[32] C. W. Reynolds, "Flocks, herds and schools: A distributed behavioral model," ACM SIGGRAPH computer graphics, vol. 21, no. 4, pp. 2534, 1987.

[33] G. Vásárhelyi, C. Virágh, G. Somorjai, N. Tarcai, T. Szörényi, T. Nepusz, and T. Vicsek, "Outdoor flocking and formation flight with autonomous aerial robots," in 2014 IEEE/RSI Int. Conf. on Intelligent Robots and Systems, Sept 2014, pp. 3866-3873.

[34] D. Pianini, M. Viroli, and J. Beal, "Protelis: Practical aggregate programming," in Proceedings of the 30th Annual ACM Symposium on Applied Computing, ser. SAC '15. New York, NY, USA: ACM, 2015, pp. 1846-1853. [Online]. Available: http://doi.acm.org/ $10.1145 / 2695664.2695913$

[35] G. Anders, A. Schiendorfer, F. Siefert, J.-P. Steghöfer, and W. Reif, "Cooperative Resource Allocation in Open Systems of Systems," ACM Trans. Auton. Adapt. Syst., vol. 10, no. 2, pp. 11:1-11:44, Jun. 2015. [Online]. Available: http://doi.acm.org/10.1145/2700323

[36] F. Fioretto, E. Pontelli, and W. Yeoh, "Distributed constraint optimization problems and applications: A survey," CoRR, vol. abs/1602.06347, 2016. [Online]. Available: http://arxiv.org/abs/1602. 06347

[37] A. Angerer, A. Hoffmann, A. Schierl, M. Vistein, and W. Reif, "Robotics api: Object-oriented software development for industrial robots," Journ. of Software Engineering for Robotics, vol. 4, no. 1, pp. 1-22, 2013.

[38] B. Wolf, C. Chwala, B. Fersch, J. Garvelmann, W. Junkermann, M. Zeeman, A. Angerer, B. Adler, C. Beck, C. Brosy et al., "The scalex campaign: scale-crossing land-surface and boundary layer processes in the tereno-prealpine observatory," Bulletin of the American Meteorological Society, no. 2016, 2016.

[39] O. Kosak, C. Wanninger, A. Angerer, A. Hoffmann, A. Schierl, and H. Seebach, "Decentralized coordination of heterogeneous ensembles using jadex," in 2016 IEEE 1st Int. Workshops on Found. and Applications of Self* Systems (FAS*W), Sept 2016, pp. 271-272.

[40] O. Kosak, G. Anders, F. Siefert, and W. Reif, "An Approach to Robust Resource Allocation in Large-Scale Systems of Systems," in SelfAdaptive and Self-Organizing Systems (SASO), 2015 IEEE 9th Int. Conf. on, Sept 2015, pp. 1-10.

[41] H. Hamann, Y. Khaluf, J. Botev, M. Divband Soorati, E. Ferrante, O. Kosak, J.-M. Montanier, S. Mostaghim, R. Redpath, J. Timmis, F. Veenstra, M. Wahby, and A. Zamuda, "Hybrid societies: Challenges and perspectives in the design of collective behavior in self-organizing systems," Frontiers in Robotics and AI, vol. 3, p. 14, 2016. [Online]. Available: http://journal.frontiersin.org/article/ 10.3389/frobt.2016.00014

[42] B. P. Gerkey and M. J. Matarić, "A formal analysis and taxonomy of task allocation in multi-robot systems," The Int. Journ. of Robotics Research, vol. 23, no. 9, pp. 939-954, 2004. 